\title{
Alterações comportamentais após cirurgia bariátrica: Uma revisão sistemática de
}

\section{literatura}

\author{
Behavioral changes after bariatric surgery: A systematic literature review \\ Cambios de comportamiento después de la cirugía bariátrica: Revisión de la literatura sistemática
}

Recebido: 12/05/2021 | Revisado: 18/05/2021 | Aceito: 20/05/2021 | Publicado: 06/06/2021

\author{
Ana Claudia Lunelli Moro \\ ORCID: https://orcid.org/0000-0001-6315-6844 \\ Universidade Alto Vale do Rio do Peixe, Brasil \\ E-mail: analunellimoro@hotmail.com \\ Ricelli Endrigo Ruppel da Rocha \\ ORCID: https://orcid.org/0000-0002-4277-1407 \\ Universidade Alto Vale do Rio do Peixe, Brasil \\ E-mail: ricellie@uniarp.edu.br
}

\begin{abstract}
Resumo
O objetivo desta revisão foi sintetizar as evidencias na literatura sobre as alterações comportamentais de pacientes obesos após cirurgia bariátrica. Foi realizada uma busca nas bases da Biblioteca Virtual de Saúde (BVS) que inclui LILACS, MEDLINE, MEDCARIB, OPAS/OMS, PAHO e WHOLIS; PubMed; EBSCO; SciELO e Scopus no período entre janeiro de 2010 a dezembro de 2020. Para a busca utilizou-se a combinação dos seguintes termos: "Cirurgia Bariátrica" OR "Bariatric Surgery" AND "Comportamento Alimentar" OR "Feeding Behavior". Foram incluídos estudos originais, desfecho principal as alterações comportamentais após cirurgia bariátrica em pacientes obesos, escritos nos idiomas português e inglês. A analise final foi composta por 13 artigos. Desses, 84,6\% foram realizados nos últimos 5 anos, $61,5 \%$ dos estudos utilizaram os delineamentos longitudinais, predominou pacientes obesos com o Índice de Massa Corporal (IMC) $\geq 35 \mathrm{~kg} / \mathrm{m}^{2}$, faixa etária de 31 a 52 anos e a maioria dos estudos foram realizados no Brasil 30,7\%, Israel 15,4\% e Reino Unido (15,4\%). As alterações no comportamento alimentar após cirurgia bariátrica foram um aumento de comedores de lanches e de doces, intolerância alimentar e palatabilidade alterada, dificuldade de ingerir alimentos proteicos e gordurosos. No comportamento emocional houve alteração do humor, aumento e/ou redução de sintomas depressivos e de ansiedade, e aumento da impulsividade. Em conclusão, após cirurgia bariátrica os pacientes obesos apresentam alterações de comportamento alimentar e emocional.
\end{abstract}

Palavras-chave: Obesidade; Cirurgia bariátrica; Comportamento alimentar.

\begin{abstract}
The goal of this review was to synthesize the evidence in the literature about the behavioral changes in obese patients after bariatric surgery. A search was carried out on the bases of the Virtual Health Library (VHL) which includes LILACS, MEDLINE, MEDCARIB, P.A.H.O. / W.H.O. (World Healht Organization), PAHO (Pan American Health Organization) and W.H.O.L.I.S. (World Health Organization Library Information System); PubMed; EBSCO; SciELO and Scopus from January 2010 to December 2020. For this search, the combination of the following terms was used: "Bariatric Surgery" OR "Bariatric Surgery" AND "Feeding Behavior" OR "Feeding Behavior". The original studies were included, the main outcome, the behavioral changes after bariatric surgery in obese patients, written in Portuguese and English. The final analysis was composed of 13 articles. Among these artciles, $84.6 \%$ were performed in the last 5 years, $61.5 \%$ of the studies used longitudinal designs, predominantly obese patients with the Body Mass Index (IMC) $\geq 35 \mathrm{~kg} / \mathrm{m}^{2}$, age range 31 to 52 years and most studies were carried out in Brazil $30.7 \%$, Israel $15.4 \%$ and the United Kingdom (15.4\%). The changes in eating behavior after bariatric surgery were an increase in snack and candy eaters, food intolerance and altered palatability, difficulty in eating protein and fatty foods. Related to the emotional behavior, there was a change in mood, an increase and / or reduction in depressive and anxiety symptoms, and an increase in impulsivity. As a conclusion, after bariatric surgery, obese patients show changes in eating and emotional behavior.
\end{abstract}

Keywords: Obesity; Bariatric surgery; Feeding behavior.

\section{Resumen}

El objetivo de esta revisión fue sintetizar la evidencia en la literatura sobre los cambios conductuales en pacientes obesos después de la cirugía bariátrica. Se realizó una búsqueda en las bases de la Biblioteca Virtual en Salud (BVS) que incluye LILACS, MEDLINE, MEDCARIB, O.P.S. / O.M.S., O.P.S. (Organización Panamericana de la Salud) y W.H.O.L.I.S. (Sistema de información bibliotecaria de la Organización Mundial de la Salud); PubMed; EBSCO; SciELO y Scopus en el período comprendido entre enero de 2010 y diciembre de 2020. Para la búsqueda, fue utilizada 
la combinación de los siguientes términos: "Cirugía bariátrica" O "Cirugía bariátrica" y "Comportamiento de alimentación" O "Comportamiento de alimentación". Se incluyeron los estudios originales, cuyo resultado principal fueron los cambios de comportamiento después de la cirugía bariátrica en pacientes obesos y que fueron escritos en portugués e inglés. El análisis final estuvo compuesto por 13 artículos. De estos artículos, el 84,6\% fueron realizados en los últimos 5 años, el $61,5 \%$ de los estudios utilizaron diseños longitudinales y predominó: pacientes obesos con el Índice de Masa Corporal (IMC) $\geq 35 \mathrm{~kg} / \mathrm{m}^{2}$, rango de edad de 31 a 52 años y la mayoría de los estudios fueron realizados en Brasil 30,7\%, Israel 15,4\% y Reino Unido (15,4\%). Los cambios en la conducta alimentaria después de la cirugía bariátrica fueron un aumento en los consumidores de bocadillos y dulces, intolerancia a los alimentos y alteración de la palatabilidad, dificultad para ingerir proteínas y alimentos grasos. En el comportamiento emocional, hubo un cambio de humor, un aumento y / o reducción de los síntomas depresivos y de ansiedad, y un aumento de la impulsividad. En conclusión de este tema, después de la cirugía bariátrica, los pacientes obesos muestran cambios en la conducta alimentaria y emocional.

Palabras clave: Obesidad; Cirugía bariátrica; Comportamiento alimenticio.

\section{Introdução}

A epidemia da obesidade é um dos maiores problemas de saúde pública no mundo e os estudos apontam que em 2025, a estimativa será de 2,3 bilhões de adultos com excesso de peso, e destes, 700 milhões apresentarão obesidade (ABESO, 2020). O número de mortes anualmente causado pela obesidade pode chegar a 2,5 milhões de pessoas, sobrecarregando o sistema público de saúde e aumentando o gasto para o tratamento das comorbidades (Sivas et al., 2020).

As principais comorbidades associadas a obesidade são o Diabetes Mellitus do Tipo 2, hipertensão, doenças cardiovasculares, dislipidemias, depressão e ansiedade, que pode causar sérios prejuízos a vida cotidiana das pessoas, impactando negativamente na qualidade de vida e aumentando prematuramente o risco de morte devido as complicações destas doenças (Kolotkin \& Andersen, 2017; Nanchahal et al., 2012)

Para o tratamento da obesidade são utilizadas estratégias como a mudança do estilo de vida através da restrição dietética e o aumento da atividade física diária, o uso de medicamentos e para alguns casos a cirurgia bariátrica (Bray, Frühbeck, Ryan, \& Wilding, 2016). A cirurgia bariátrica é composta por um grupo de intervenções cirúrgicas com o objetivo de limitar a capacidade do corpo em consumir e absorver os alimentos através da redução e/ou comprimento do estomago e do intestino (Gilmartin, Bath-Hextall, Maclean, Stanton, \& Soldin, 2016). É a intervenção com melhores resultados para a perda e manutenção do peso corporal em pacientes com obesidade mórbida (Índice de Massa Corporal $\geq 40 \mathrm{~kg} / \mathrm{m}^{2} \mathrm{ou} \geq 35 \mathrm{~kg} / \mathrm{m}^{2} \mathrm{com}$ comorbidades), sendo considerada a terapia mais efetiva para o controle da obesidade (Lupoli et al., 2017).

Contudo, após a Cirurgia Bariátrica ocorrem mudanças na anatomia e na função gastrointestinal, diminuindo a ingestão de vitaminas e micronutrientes, dificultando a absorção que ao longo tempo podem causar complicações tais como anemia, osteoporose, má nutrição, distúrbios neurológicos e comportamentais (Castanha et al., 2018; Mesureur \& Arvanitakis, 2017). Dentre os distúrbios comportamentais mais frequentes estão a anorexia e bulimia nervosa, a perda do controle alimentar, compulsão alimentar e mudanças no estado de humor e na qualidade de vida relacionada à saúde (Brode \& Mitchell, 2019).

Apesar das mudanças comportamentais que as pesquisas mostram após a realização da cirurgia bariátrica, não foram encontradas revisões bibliográficas da literatura sobre o tema. Diante disso, esta pesquisa trará uma visão atual do problema e contribuíra para uma atualização científica dentro desse campo, possibilitando aos profissionais da saúde informações relevantes para o planejamento de medidas e ações que atenuem os distúrbios comportamentais em pacientes submetidos a cirurgia bariátrica.

Portanto, o objetivo desta pesquisa foi sintetizar as evidencias na literatura sobre as alterações comportamentais de pacientes obesos após cirurgia bariátrica. 


\section{Metodologia}

Esta revisão sistemática utilizou os procedimentos metodológicos estabelecidos pelo guia Preferred Reporting Items for Systematic Reviews and Meta-Analyses - PRISMA (Page et al., 2021). Para a busca sistemática e as definições dos descritores, optou-se por utilizar os Descritores em Ciências da Saúde (DeCS). Dessa maneira, os termos encontrados para a realização desta pesquisa foram testados e por fim chegou-se a seguinte combinação nos idiomas português e inglês: "cirurgia bariátrica” OR "bariatric surgery" AND “comportamento alimentar” OR "feeding behavior”.

A busca foi realizada nos últimos dez anos que antecederam a pesquisa em cinco (05) bases de dados: Biblioteca Virtual de Saúde (BVS) que inclui LILACS, MEDLINE, MEDCARIB, OPAS/OMS, PAHO e WHOLIS; PubMed; EBSCO; SciELO e Scopus. A consulta as bases de dados iniciaram na primeira semana de setembro de 2020 e finalizou na última semana de setembro de 2020.

Para a inclusão dos artigos foram estabelecidos os seguintes critérios: a) Estudos originais; b) Considerando como desfecho principal as alterações comportamentais após cirurgia bariátrica em pacientes obesos; c) Idiomas: português e inglês; e) Publicados a partir de janeiro de 2010 a dezembro de 2020. Foram excluídos os estudos de revisão (narrativas, sistemáticas e/ou meta análises), dissertações, teses, relatórios e monografias. Dois pesquisadores realizaram de maneira independente cada etapa do processo de revisão e caso houvesse divergência no processo de inclusão e exclusão, foi realizada uma reunião de consenso entre os pesquisadores.

Inicialmente, todos os títulos selecionados nas bases de dados $(n=164)$ foram transferidos para o software EndNote e, em seguida, foram excluídos os títulos repetidos $(n=43)$. O processo de revisão sistemática foi composto por quatro fases: A primeira fase consistiu na leitura dos títulos, dos 121 (100\%) títulos encontrados, 35 artigos (28,9\%) foram excluídos por não apresentarem relação com o tema, publicados em outra língua e outros documentos como monografias e dissertações. Na segunda fase foi realizada a leitura dos resumos $(n=86 ; 71,0 \%)$, destes 43 artigos foram excluídos por não ter avaliado após a cirurgia bariátrica o comportamento alimentar ou algum tipo de alteração comportamental. Na terceira fase foi realizada a leitura na íntegra dos artigos restantes $(n=43 ; 35,5 \%)$ e destes, 30 artigos foram excluídos por não apresentar como desfecho principal as alterações comportamentais após cirurgia bariátrica. Ao final, 13 artigos (10,7\%) atenderam aos critérios empregados e foram selecionados para revisão. A Figura 1 apresenta o processo de busca aos artigos, os resultados e os respectivos motivos de exclusão dos mesmos. 
Figura 1: Fluxograma de seleção dos artigos.
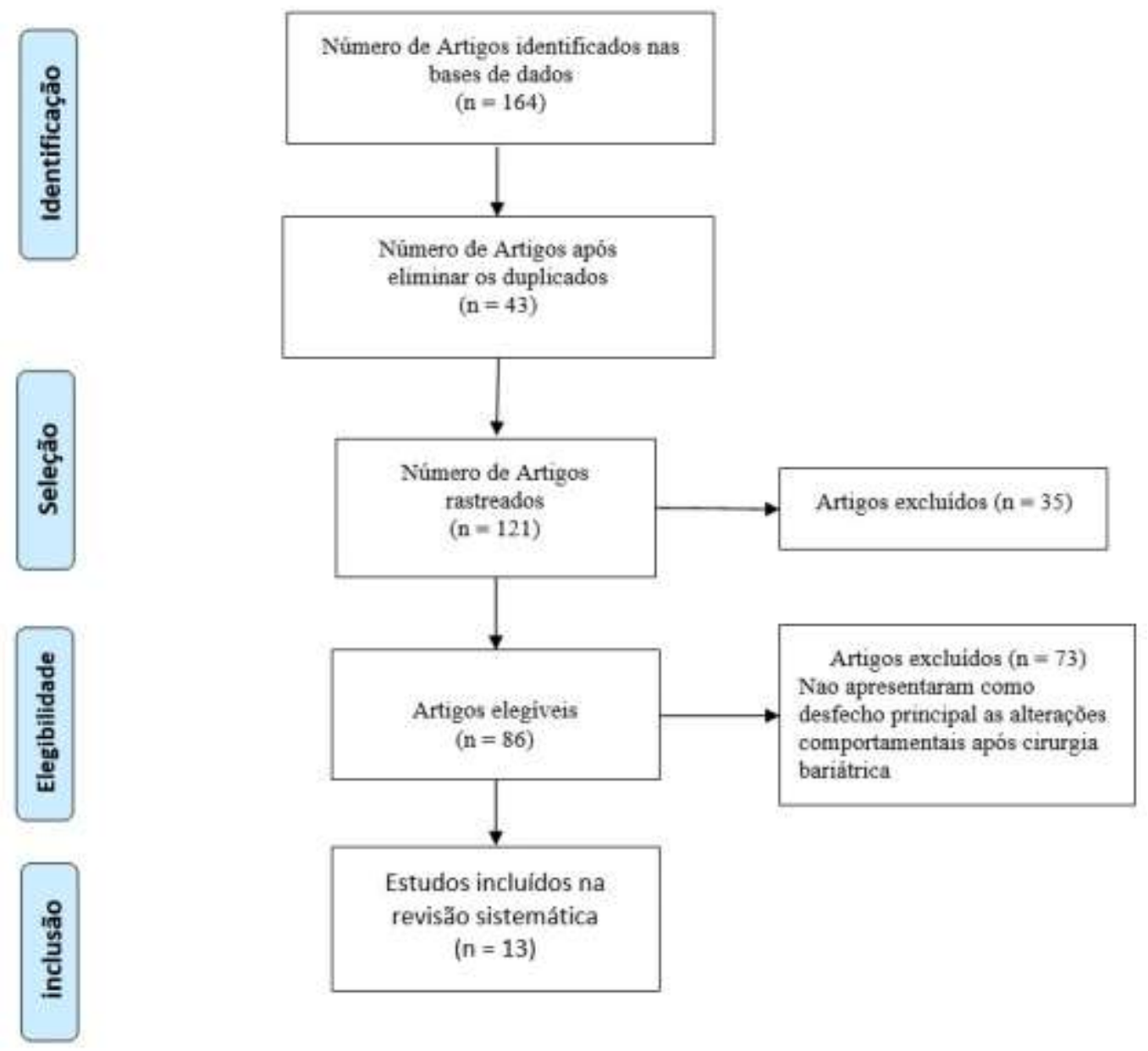

Fonte: Autores.

\section{Resultados}

No total dos 13 artigos selecionados para a revisão sistemática, 84,6\% ( $\mathrm{n}=11)$ foram realizados nos últimos 5 anos. Os delineamentos longitudinais predominaram em $61,5 \%$ dos estudos e os pacientes obesos com o Índice de Massa Corporal (IMC) $\geq 35 \mathrm{~kg} / \mathrm{m}^{2}$ foram os mais frequentes que realizaram a cirurgia bariátrica (Tabela 1). A faixa de idade dos pacientes obesos que fizeram a cirurgia bariátrica foi de 31 a 52 anos e a maioria dos estudos foram realizados no Brasil 30,7\% ( $\mathrm{n}=4$ ), Israel $15,4 \%(\mathrm{n}=2)$ e Reino Unido $(15,4 \%(\mathrm{n}=2)$.

As principais alterações após cirurgia bariátrica foram relacionadas aos comportamentos alimentares e emocionais (Tabela 1). No comportamento alimentar houve um aumento de comedores de lanches e de doces, intolerância alimentar e palatabilidade alterada, dificuldade de ingerir alimentos proteicos e gordurosos. Com relação ao comportamento emocional após cirurgia bariátrica houve alteração do humor, aumento e/ou redução de sintomas depressivos e de ansiedade e aumento da impulsividade.

Além disso, também foi verificado outras alterações após cirurgia bariátrica como a falta de energia, cansaço, alterações do sono, anemia, redução de pensamentos hedônicos, ganho de peso, entre outros (Tabela 1). 
Tabela 1: Caraterísticas e resultados dos estudos incluídos na revisão.

\begin{tabular}{ccccc}
\hline Autores & Delineamento & Amostra & Idade & País \\
\hline $\begin{array}{c}\text { Dos Rodrigues, de } \\
\text { Vasconcelos, \& } \\
\text { Gomes, 2020 }\end{array}$ & Transversal & $\begin{array}{c}\text { 44 mulheres obesas } \\
\text { com IMC } \geq 40 \mathrm{~kg} \cdot \mathrm{m}^{2}\end{array}$ & 18 a 59 anos & Brasil \\
& & & & \\
$\begin{array}{l}\text { Susmallian, } \\
\text { Nikiforova, }\end{array}$ & Longitudinal & $\begin{array}{c}300 \text { pacientes obesos } \\
\text { com IMC } \geq 40 \mathrm{~kg} \cdot \mathrm{m}^{2}\end{array}$ & 18 anos ou mais & Israel \\
$\begin{array}{c}\text { Azoulai, \& Barnea, } \\
\text { 2019 }\end{array}$ & & &
\end{tabular}
2019

acientes obesos

(2)

\begin{tabular}{|c|c|c|c|c|c|}
\hline $\begin{array}{c}\text { Bressan \& Trevisol, } \\
2019\end{array}$ & Transversal & 71 pacientes obesos & 19 a 66 anos & Brasil & $\begin{array}{ll}\text { - } & \text { Falta de energia } \\
\text { - } & \text { Cansaço } \\
\text { - } & \text { Alterações do sono e do apetite } \\
\text { - } & \text { Anemia } \\
\end{array}$ \\
\hline $\begin{array}{l}\text { Nikiforova, Barnea, } \\
\text { Azulai, \& } \\
\text { Susmallian, } 2019\end{array}$ & Longitudinal & 300 pacientes obesos & 18 anos ou mais & Israel & $\begin{array}{l}\text { - Aumento significativo de comedores } \\
\text { de lanches e comedores de doces } \\
\text { - } \quad \text { Piora dos hábitos alimentares }\end{array}$ \\
\hline $\begin{array}{l}\text { Al Khalifa \& Al } \\
\text { Ansari, } 2018\end{array}$ & Retrospectivo & $\begin{array}{l}84 \text { pacientes obesos } \\
\text { com } \mathrm{IMC} \geq 40 \mathrm{~kg} \cdot \mathrm{m}^{2}\end{array}$ & $\begin{array}{l}35,8 \text { anos a } 36,1 \\
\text { anos }\end{array}$ & Barein & $\begin{array}{l}\text { - Apresentaram intolerância alimentar } \\
\text { - Comportamento alimentar } \\
\text { transtornado } \\
\end{array}$ \\
\hline $\begin{array}{l}\text { Paixão, Lourenço, } \\
\text { Dias, \& Nogueira, } \\
2018\end{array}$ & Transversal & $\begin{array}{l}42 \text { pacientes obesos } \\
\text { com IMC } \geq 40 \mathrm{~kg} \cdot \mathrm{m}^{2}\end{array}$ & 20 a 59 anos & Brasil & $\begin{array}{l}\text { - Após cirurgia aumentou a } \\
\text { dificuldade de ingerir alimentos } \\
\text { como carnes vermelhas, pães e } \\
\text { massas }\end{array}$ \\
\hline $\begin{array}{l}\text { Nance, Eagon, } \\
\text { Klein, \& Pepino, } \\
2017\end{array}$ & Longitudinal & $\begin{array}{l}31 \text { pacientes obesos } \\
\text { com } \mathrm{IMC} \geq 40 \mathrm{~kg} \cdot \mathrm{m}^{2}\end{array}$ & $\begin{array}{l}36,6 \text { anos a } 43,0 \\
\text { anos }\end{array}$ & EUA & $\begin{array}{l}\text { - } \quad \text { Redução da frequência do desejo de } \\
\text { se alimentar } \\
\text { - Alteração da palatabilidade do doce } \\
\text { de agradável para desagradável }\end{array}$ \\
\hline $\begin{array}{l}\text { Kanerva, Larsson, } \\
\text { Peltonen, Lindroos, } \\
\text { \& Carlsson, } 2017\end{array}$ & Longitudinal & $\begin{array}{l}2010 \text { pacientes obesos } \\
\text { com IMC } \geq 34 \mathrm{~kg} \cdot \mathrm{m}^{2} \\
\text { para homens e IMC } \geq \\
38 \mathrm{~kg} \cdot \mathrm{m}^{2} \text { para mulheres }\end{array}$ & $\begin{array}{l}\text { Média de } 34 \\
\text { anos para } \\
\text { homens e } 38 \\
\text { anos para } \\
\text { mulheres }\end{array}$ & Suécia & $\begin{array}{l}\text { - Redução do consumo de } \\
\text { carboidratos, proteínas e gorduras }\end{array}$ \\
\hline Schag et al., 2016 & Longitudinal & $\begin{array}{l}65 \text { pacientes obesos } \\
\text { com } \mathrm{IMC} \geq 35 \mathrm{~kg} \cdot \mathrm{m}^{2}\end{array}$ & $\begin{array}{l}\text { Média de 49,0 } \\
\text { anos }\end{array}$ & Alemanha & $\begin{array}{l}\text { - Aumentou os sintomas de depressão } \\
\text { e impulsividade comprometendo a } \\
\text { persa de peso }\end{array}$ \\
\hline $\begin{array}{l}\text { Husted \& Ogden, } \\
\qquad 2014\end{array}$ & Longitudinal & $\begin{array}{l}91 \text { pacientes obesos } \\
\text { com } \mathrm{IMC} \geq 35 \mathrm{~kg} \cdot \mathrm{m}^{2}\end{array}$ & $\begin{array}{l}\text { Média de } 43,1 \\
\text { anos }\end{array}$ & $\begin{array}{l}\text { Reino } \\
\text { Unido }\end{array}$ & 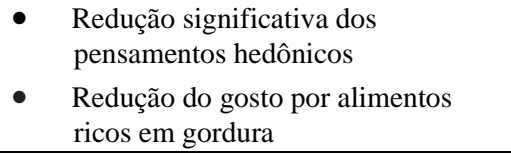 \\
\hline $\begin{array}{l}\text { Konttinen, } \\
\text { Peltonen, Sjöström, } \\
\text { Carlsson, \& } \\
\text { Karlsson, 2015 }\end{array}$ & Transversal & $\begin{array}{l}2010 \text { pacientes obesos } \\
\text { com IMC } \geq 34 \mathrm{~kg} \cdot \mathrm{m}^{2} \\
\text { para homens e IMC } \geq \\
38 \mathrm{~kg} \cdot \mathrm{m}^{2} \text { para mulheres }\end{array}$ & 37 a 60 anos & Noruega & $\begin{array}{l}\text { Apresentaram comportamento } \\
\text { alimentar de comer emocional, } \\
\text { comer descontrolado e ansiedade }\end{array}$ \\
\hline $\begin{array}{c}\text { Guthrie, Tetley, \& } \\
\text { Hill, } 2014\end{array}$ & Longitudinal & $\begin{array}{l}21 \text { mulheres com IMC } \geq \\
25 \mathrm{~kg} \cdot \mathrm{m}^{2}\end{array}$ & $\begin{array}{l}\text { Média de } 43,8 \\
\text { anos }\end{array}$ & $\begin{array}{l}\text { Reino } \\
\text { Unido }\end{array}$ & $\begin{array}{l}\text { Inicialmente aumento o desejo por } \\
\text { alimentos salgados e depois por } \\
\text { alimentos doces }\end{array}$ \\
\hline Tae et al., 2014 & Longitudinal & $\begin{array}{l}32 \text { mulheres obesas } \\
\text { com IMC } \geq 35 \mathrm{~kg} \cdot \mathrm{m}^{2}\end{array}$ & $\begin{array}{l}\text { Média de } 41,0 \\
\text { anos }\end{array}$ & Brasil & $\begin{array}{l}\text { - } \begin{array}{l}\text { Redução dos sintomas depressivos e } \\
\text { ansiosos }\end{array} \\
\text { Redução do comportamento } \\
\text { bulímico } \\
\text { - } \\
\begin{array}{l}\text { Diminuição do uso de } \\
\text { antidepressivos e inibidores de } \\
\text { apetite }\end{array}\end{array}$ \\
\hline
\end{tabular}




\section{Discussão}

A maioria dos estudos foram realizados nos últimos 5 anos que antecederam a presente pesquisa e os delineamentos longitudinais foram os mais utilizados (Tabela 1). Estes achados podem estar relacionados com o aumento nos últimos anos da obesidade no mundo e com o aperfeiçoamento das técnicas cirúrgicas, possibilitando um número maior de pacientes obesos que buscam por tratamentos para redução do peso corporal, principalmente a cirurgia bariátrica que é o método mais efetivo para a perda de peso (Hales, 2020). Além disso, o delineamento longitudinal acompanha os sujeitos ao longo do tempo para verificar possíveis efeitos de determinadas intervenções, o que pode explicar a razão dos pesquisadores para a sua utilização nos estudos com cirurgia bariátrica (Mota, 2010).

A cirurgia bariátrica foi realizada principalmente em mulheres com idade acima dos 30 anos (Tabela 1). A razão disso, pode estar relacionada a maior prevalência de mulheres com obesidade no mundo e a imposição da sociedade para um corpo ideal. Com relação as taxas de obesidade, nas mulheres ocorrem em $15 \%$ da população mundial enquanto que nos homens esta taxa baixa para $11 \%$, o que pode explicar a maior busca pela cirurgia bariátrica no sexo feminino (Carvalho \& Rosa, 2019). O corpo ideal que a sociedade impõe nas mulheres tem aumentado os procedimentos cirúrgicos cada vez mais na população jovem feminina (Tonatto-Filho, Gallotti, Chedid, Grezzana-Filho, \& Garcia, 2019; Bressan \& Schuelter-Trevisol, 2019).

Isto pode ser observado em um estudo de revisão sistemática realizado entre 1999 a 2014, com o objetivo de descrever o perfil antropométrico e de comorbidades em pacientes submetidos à cirurgia bariátrica pelo Sistema Único de Saúde no Brasil (SUS), mostrando que $79 \%$ das cirurgias foram realizadas pelo sexo feminino e somente $21 \%$ no sexo masculino (Kelles, Diniz, Machado, \& Barreto, 2015). Em outro estudo que avaliou entre janeiro de 2010 e dezembro de 2016, foram observadas 46.035 hospitalizações para realização de cirurgia bariátrica pelo SUS e destes $85,4 \%$ foram realizados em mulheres (Carvalho \& Rosa, 2019).

A cirurgia bariátrica foi realizada principalmente em pacientes obesos com IMC $\geq 35 \mathrm{~kg} \cdot \mathrm{m}^{2}$ (Tabela 1). A cirurgia bariátrica é indicada para pacientes com IMC maior que $40 \mathrm{~kg} . \mathrm{m}^{2}$ ou IMC maior ou igual a $35 \mathrm{~kg} . \mathrm{m}^{2}$ associado com comorbidades (Zilberstein, Santo, \& Carvalho, 2019). É importante salientar que o IMC maior ou igual a $30 \mathrm{~kg} \cdot \mathrm{m}^{2}$ é considerado um fator de risco para o desenvolvimento do Diabetes Mellitus do tipo 2 (DM2), Hipertensão Arterial Sistêmica (HAS), dislipidemia e apneia obstrutiva do sono (AOS), câncer, entre outras doenças, aumentando a probabilidade de morte prematura (Husted \& Ogden, 2014).

Nesta revisão sistemática, o Brasil foi o país com maior número de estudos que avaliaram as alterações comportamentais após cirurgia bariátrica em pacientes obesos (Tabela 1). Inferimos que este resultado pode ser explicado pelo aumento do número de cirurgias bariátricas nos últimos 10 anos no país. De acordo com a Sociedade Brasileira de Cirurgia Bariátrica e Metabólica (2016), o Brasil é o segundo país com maior número de cirurgias bariátricas, ficando atrás apenas dos Estados Unidos. A quantidade destas cirurgias entre brasileiros teve um crescimento de 72 mil em 2012 para 100 mil em 2016. Estima-se que o aumento no período de 2006 a 2015 tenha sido de 300\% (Zilberstein et al., 2019).

Os estudos mostraram que os pacientes obesos após a cirurgia bariátrica apresentaram alterações no comportamento alimentar, dentre os principais estão um aumento da ingestão de lanches e de doces, dificuldade de ingerir alimentos proteicos e gordurosos, intolerância alimentar e palatabilidade alterada (Tabela 1). As alterações no comportamento alimentar após cirurgia bariátrica são observadas em 30\% a 50\% dos obesos (Rutledge, Adler, \& Friedman, 2011; Tae et al., 2014). O período imediato após a cirurgia é o mais difícil e desconfortável para os pacientes, devido as várias tentativas de adaptação à nova dieta, ao aumento da expectativa, ansiedade e insegurança relativa à nova situação (Bressan \& Schuelter-Trevisol, 2019). Esta condição pode resultar na perda sobre o controle dos alimentos, desenvolver vícios alimentares e induzir o consumo de alimentos ricos em gordura, açúcares e/ou sal, como os lanches e doces (Susmallian et al., 2019; Benzerouk et al. 2018). 
Também foi verificado nos estudos que determinadas alterações emocionais como do humor, aumento e/ou redução de sintomas depressivos e de ansiedade e aumento da impulsividade, ocorreram após a cirurgia bariátrica nos pacientes obesos (Tabela 1). A cirurgia bariátrica tende a provocar déficits nutricionais importantes como a deficiência de vitaminas do complexo B que podem desenvolver diversos problemas como anemias, neuropatias e sintomas psiquiátricos, especialmente transtornos depressivos e de humor (Bressan \& Schuelter-Trevisol, 2019). Destacamos que as alterações do estado de humor e do estado depressivo de muitos obesos após cirurgia bariátrica também muda o padrão dos hábitos alimentares e o ganho de peso, pois indivíduos deprimidos têm por carboidratos, gorduras e doces um alimento rico que parece resultar da melhora no humor entretanto, são alimentos ricos em energia aumentando consumo energético e facilitando o retorno do peso perdido (Lazarevich et al. ,2016)

Apesar da importância do estudo, não foram apresentadas as alterações comportamentais de acordo com os diferentes métodos de cirurgia bariátrica, limitando os resultados. Outra limitação que deve ser apontada neste estudo foi a não identificação das mudanças comportamentais que ocorreram de acordo com os períodos avaliados nos estudos.

\section{Conclusão}

Em resumo, os pacientes obesos que realizaram a cirurgia bariátrica apresentaram alterações de comportamento alimentar e de comportamento emocional após a intervenção. As alterações no padrão alimentar e o déficit de nutrientes após a cirurgia bariátrica são apontados nos estudos como os principais fatores para o desenvolvimento de alterações comportamentais.

Sugerimos que os obesos que realizam a cirurgia bariátrica sejam acompanhados por nutricionais e psicólogos para diminuir os agravos à saúde física e mental e continuar a perda do peso corporal ao longo do tempo. Salientamos ainda que mais pesquisas precisam ser realizadas para analisar as alterações comportamentais que podem ocorrer dependendo do método cirúrgico que os pacientes obesos realizam para buscar intervenções específicas.

\section{Referências}

ABESO. Obesidade e síndrome metabólica: mapa da obesidade. Associação Brasileira para o Estudo da Obesidade e da Síndrome Metabólica. Retrieved from https://abeso.org.br/obesidade-e-sindrome-metabolica/mapa-da-obesidade/.

Al Khalifa, K., \& Al Ansari, A. (2018). Quality of life, food tolerance, and eating disorder behavior after laparoscopic gastric banding and sleeve gastrectomy - results from a middle eastern center of excellence. BMC Obes, 5, 44. 10.1186/s40608-018-0220-6

Benzerouk F, Gierski F, Ducluzeau PH, Bourbao-Tournois C, Gaubil-Kaladjian I, Bertin E' et al. (2018). Food addiction, in obese patients seeking bariatric surgery, is associated with higher prevalence of current mood and anxiety disorders and past mood disorders. Psychiatry Res. 2018 ; 267: 473-479. org/10.1016/j.psychres.2018.05.087 PMID: 29980127

Bray, G. A., Frühbeck, G., Ryan, D. H., \& Wilding, J. P. (2016). Management of obesity. Lancet, 387(10031), 1947-1956. 10.1016/s0140-6736(16)00271-3

Bressan, J. de A., \& Schuelter-Trevisol, F. (2019). Avaliação da autoestima e depressão após cirurgia bariátrica. Revista Brasileira de Obesidade, Nutrição e Emagrecimento, 13(79), 446-456. http://www.rbone.com.br/index.php/rbone/article/view/988

Brode, C. S., \& Mitchell, J. E. (2019). Problematic Eating Behaviors and Eating Disorders Associated with Bariatric Surgery. Psychiatr Clin North Am, 42(2), 287-297. 10.1016/j.psc.2019.01.014

Carvalho, A. d. S., \& Rosa, R. d. S. (2019). Cirurgias bariátricas realizadas pelo Sistema Único de Saúde no período 2010-2016: estudo descritivo das hospitalizações no Brasil. Epidemiologia e Serviços de Saúde, 28(1). 10.5123/s1679-49742019000100023

Castanha, C. R., TCBC-PE, Á. A. B. F., Castanha, A. R., Belo, G. Q. M. B., Lacerda, R. M. R., \& Vilar, L. (2018). Avaliação da qualidade de vida, perda de peso e comorbidades de pacientes submetidos à cirurgia bariátrica. Revista do Colégio Brasileiro de Cirurgiões, 45(3), e1864. doi.org/10.1590/0100-6991e20181864

Dos Rodrigues, L. S., de Vasconcelos, P. H. C., \& Gomes, D. L. (2020). Weight regain and eating behavior in physically active and inactive women after 24 months of bariatric surgery. Eat Weight Disord. 10.1007/s40519-020-00973-w

Gilmartin, J., Bath-Hextall, F., Maclean, J., Stanton, W., \& Soldin, M. (2016). Quality of life among adults following bariatric and body contouring surgery: a systematic review. JBI Database System Rev Implement Rep, 14(11), 240-270. 10.11124/jbisrir-2016-003182 
Guthrie, H., Tetley, D., \& Hill, A. J. (2014). Quasi-prospective, real-life monitoring of food craving post-bariatric surgery: comparison with overweight and normal weight women. Clin Obes, 4(3), 136-142. 10.1111/cob.12054

Hales CM, C. M., Fryar CD, Ogden CL. (2020). Prevalence of obesity and severe obesity among adults: United States, 2017-2018. National Center for Health Statistics (360). Retrieved from https://www.cdc.gov/nchs/products/databriefs/db360.htm\#References

Husted, M., \& Ogden, J. (2014). Emphasising Personal Investment Effects Weight Loss and Hedonic Thoughts about Food after Obesity Surgery. Journal of Obesity, 2014, 810374. 10.1155/2014/810374

Kanerva, N., Larsson, I., Peltonen, M., Lindroos, A. K., \& Carlsson, L. M. (2017). Changes in total energy intake and macronutrient composition after bariatric surgery predict long-term weight outcome: findings from the Swedish Obese Subjects (SOS) study. Am J Clin Nutr, 106(1), 136-145. 10.3945/ajcn.116.149112

Kelles, S. M. B., Diniz, M. d. F. H. S., Machado, C. J., \& Barreto, S. M. (2015). Perfil de pacientes submetidos à cirurgia bariátrica, assistidos pelo Sistema Único de Saúde do Brasil: revisão sistemática. Cadernos de Saúde Pública, 31(8), 1587-1601. doi.org/10.1590/0102-311X00022714

Kolotkin, R. L., \& Andersen, J. R. (2017). A systematic review of reviews: exploring the relationship between obesity, weight loss and health-related quality of life. Clin Obes, 7(5), 273-289. 10.1111/cob.12203

Konttinen, H., Peltonen, M., Sjöström, L., Carlsson, L., \& Karlsson, J. (2015). Psychological aspects of eating behavior as predictors of 10-y weight changes after surgical and conventional treatment of severe obesity: results from the Swedish Obese Subjects intervention study. Am J Clin Nutr, 101(1), 16-24. 10.3945/ajcn.114.095182

Lazarevich, I., Irigoyen Camacho, M. E., Velázquez-Alva, M. D. C., \& Zepeda Zepeda, M. (2016). Relationship among obesity, depression, and emotional eating in :young adults. Appetite. 2016; 107: 639-644. org/10.1016/j.appet.2016.09.011

Lupoli, R., Lembo, E., Saldalamacchia, G., Avola, C. K., Angrisani, L., \& Capaldo, B. (2017). Bariatric surgery and long-term nutritional issues. World J Diabetes, 8(11), 464-474. 10.4239/wjd.v8.i11.464

Mesureur, L., \& Arvanitakis, M. (2017). Metabolic and nutritional complications of bariatric surgery: a review. Acta Gastroenterol Belg, 80(4), 515-525.

Mota, M. M. P. E. (2010). Metodologia de Pesquisa em Desenvolvimento Humano: Velhas Questões Revisitadas. Psicologia em Pesquisa, 4(2), $144-149$.

Nance, K., Eagon, J. C., Klein, S., \& Pepino, M. Y. (2017). Effects of Sleeve Gastrectomy vs. Roux-en-Y Gastric Bypass on Eating Behavior and Sweet Taste Perception in Subjects with Obesity. Nutrients, 10(1). 10.3390/nu10010018

Nanchahal, K., Power, T., Holdsworth, E., Hession, M., Sorhaindo, A., Griffiths, U., \& Haines, A. (2012). A pragmatic randomised controlled trial in primary care of the Camden Weight Loss (CAMWEL) programme. BMJ Open, 2(3). 10.1136/bmjopen-2011-000793

Nikiforova, I., Barnea, R., Azulai, S., \& Susmallian, S. (2019). Analysis of the Association between Eating Behaviors and Weight Loss after Laparoscopic Sleeve Gastrectomy. Obesity Facts, 12(6), 618-631. 10.1159/000502846

Page, M. J., McKenzie, J. E., Bossuyt, P. M., Boutron, I., Hoffmann, T. C., Mulrow, C. D., \& Moher, D. (2021). The PRISMA 2020 statement: an updated guideline for reporting systematic reviews. Bmj, 372(71),10.1136/bmj.n71

Paixão, A. L., Lourenço, V. V., Dias, J. S., \& Nogueira, A. A. C. (2018). Perfil alimentar de pacientes pós cirurgia bariátrica. Revista Brasileira de Obesidade, Nutrição de Emagrecimento, 12(71), 391-399.

Rutledge, T., Adler, S., \& Friedman, R. (2011). A prospective assessment of psychosocial factors among bariatric versus non-bariatric surgery candidates. Obes Surg, 21(10), 1570-1579. 10.1007/s11695-010-0287-8

Sociedade Brasileira de Cirurgia Bariatrica e Metabolica. (2016). História da cirurgia bariátrica no Brasil. Retrieved from http://www.sbcbm.org.br/wordpress/pagina-exemplo/historia-da-cirurgia-bariatrica

Schag, K., Mack, I., Giel, K. E., Ölschläger, S., Skoda, E. M., von Feilitzsch, M., \& Teufel, M. (2016). The Impact of Impulsivity on Weight Loss Four Years after Bariatric Surgery. Nutrients, 8(11). 10.3390/nu8110721

Sivas, F., Moran, M., Yurdakul, F., Ulucaköy Koçak, R., Başkan, B., \& Bodur, H. (2020). Physical activity, musculoskeletal disorders, sleep, depression, and quality of life before and after bariatric surgery. Turk J Phys Med Rehabil, 66(3), 281-290. 10.5606/tftrd.2020.3694

Susmallian, S., Nikiforova, I., Azoulai, S., \& Barnea, R. (2019). Outcomes of bariatric surgery in patients with depression disorders. PLoS One, 14(8), e0221576. 10.1371/journal.pone.0221576

Tae, B., Pelaggi, E. R., Moreira, J. G., Waisberg, J., Matos, L. L. d., \& D'Elia, G. (2014). Impact of bariatric surgery on depression and anxiety symptons, bulimic behaviors and quality of life. Revista do Colégio Brasileiro de Cirurgiões, 41(3), 155-160. doi.org/10.1590/S0100-69912014000300004

Tonatto-filho, A. J., Gallotti, F. M., Chedid, M. F., Grezzana-filho, T. D. J. M., \& Garcia, A. M. S. V. (2019). Bariatric surgery in brazilian public health system: the good, the bad and the ugly, or a long way to go. yellow sign!. Arquivos Brasileiros de Cirurgia Digestiva, 32(4), e1470. doi.org/10.1590/0102$672020190001 \mathrm{e} 1470$

Zilberstein, B., Santo, M. A., \& Carvalho, M. H. (2019). Critical analysis of surgical treatment techniques of morbid obesity. Arquivos Brasileiros de Cirurgia Digestiva (São Paulo), 32(3). 10.1590/0102-672020190001e1450 